\title{
Prediction of human microRNA hairpins using only positive sample learning
}

\author{
Dang Hung Tran ${ }^{*}, 1$, Tho Hoan Pham ${ }^{2}$, Kenji Satou ${ }^{1,3}$ \& Tu Bao Ho ${ }^{1}$ \\ ${ }^{1}$ Japan Advanced Institute of Science and Technology, 1-1 Asahidai, Nomi, Ishikawa 923-1292 Japan. ${ }^{2}$ Hanoi National University of Education, 136 Xuan \\ Thuy, Hanoi, Viet Nam. ${ }^{3}$ Kanazawa University, Kakuma, Kanazawa 920-1192, Japan. ${ }^{*}$ Correspondence should be addressed to Dang Hung Tran \\ (hungtd@jaist.ac.jp).
}

\begin{abstract}
MicroRNAs (miRNAs) are small molecular non-coding RNAs that have important roles in the post-transcriptional mechanism of animals and plants. They are commonly 21-25 nucleotides (nt) long and derived from 60-90 nt RNA hairpin structures, called miRNA hairpins. A larger number of sequence segments in the human genome have been computationally identified with such 60-90 nt hairpins, however the majority of them are not miRNA hairpins. Most existing computational methods for predicting miRNA hairpins are based on a two-class classifier to distinguish between miRNA hairpins and other sequence segments with hairpin structures. The difficulty of these methods is how to select hairpins as negative examples of miRNA hairpins in the training dataset, since only a few miRNA hairpins are available. Therefore, these classifiers may be mis-trained due to some false negative examples of the training dataset. In this paper, we introduce a one-class support vector machine (SVM) method to predict miRNA hairpins among the hairpin structures. Different from existing methods for predicting miRNA hairpins, the one-class SVM classifier is trained only on the information of the miRNA class. We also illustrate some examples of predicting miRNA hairpins in human chromosomes 10, 15, and 21, where our method overcomes the above disadvantages of existing two-class methods.
\end{abstract}

Keywords: MicroRNA; Hairpin; One-class SVM

\section{INTRODUCTION}

MicroRNAs (miRNAs) are small, non-coding RNAs (2125 nucleotides in length) that regulate the expression of protein-encoding genes at the post-transcriptional level [1, 2 , 21]. Each miRNA derives from a larger precursor, which folds into an imperfect stem-loop structure.

In human, the processing and maturation of miRNAs are divided into several steps before silencing their targets. First, the long primary transcripts (pri-miRNAs), which can be up to several kilobases, are processed by Droshacomplex in nucleus to yield precursor miRNAs (premiRNAs) [10, 12]. The pre-miRNA is a double-stranded sequence of about 60-90 nt with a 2-nt 3' overhang and forms a hairpin structure (also called miRNA hairpin). Second, pre-miRNAs are transported from the nucleus into the cytoplasm by another complex, which consists of Exportin 5 and RanGTP [6, 29]. Subsequently, the premiRNA is cleaved into an imperfect double-stranded RNA duplex by endonuclease RNase III enzyme called Dicer [25, 29, 42]. This duplex is composed of the mature miRNA strand and its complementary strand. Finally, mature miRNAs are incorporated into RICS (RNAinduced silencing complex) before they bind to their targets to regulate gene expression.

Until now, several computational approaches have been proposed for predicting miRNAs. Most of them are based on the common structural characteristic of secondary structures of their pre-miRNAs [15, 35, 40]. Since pre-miRNAs are often short (60-90 nt), there can be too many subsequences in a genome having hairpin structures. However, only a minority of them are miRNA hairpins. Using only information of their structures therefore may not allow us to distinguish miRNA hairpins from other hairpin structures. Other methods that consider information of both sequences and structures are needed.

Most methods so far used a two-class classifier to separate the miRNA hairpins from the ones assumed to be negative. The main difference between these methods is how negative examples are selected for the two-class classifier training dataset. For example, Szafranski et al. [35] and Xue et al. [40] selected examples that overlap with one of the last exon of known mRNAs; or Helvik et al. [15] tried to get them randomly from DNA sequences with hairpin structures as "negative" miRNA hairpins. The negative examples collected in such ways would contain false negatives, since no study so far has mentioned the information regarding true negative miRNA hairpins. In other words, only the information of miRNA hairpins is available. Therefore, the classifier of existing methods may be incorrect, due to some false negative miRNA hairpins contained in the training dataset.

In this paper, we present a new method for predicting miRNA hairpins that employs support vector machines 
for one-class classification (one-class SVMs). One-class SVMs recently have been successfully applied in several areas, especially domains with imbalanced data such as document classification [30], gene prediction [19] and image retrieval [9]. Different from previous methods for predicting miRNA hairpins, our method uses only available miRNA hairpins for training the model, while other methods train their classifiers by using an additional dataset of negative examples, which may contain some false negatives as explained above. Moreover, more features of hairpin sequences and structures are used to represent hairpins, with expectation that they would be useful for the model. Our one-class SVM classifier gave good results in predicting miRNA hairpins. We also illustrated some examples of predicting miRNA hairpins in human chromosomes 10, 15, and 21 where our method can avoid the problem of false negative examples of the existing two-class methods.

\section{MATERIALS AND METHODS}

\subsection{Datasets for training and testing}

As mentioned in Section 1, our method uses one-class SVMs to recognize miRNA hairpins from potential ones produced by ScorePin [15]. To do this, the one-class SVM model should capture the characteristics of known miRNA hairpins. In our work, the positive class we used consists of 474 known human miRNA hairpins from miRBase (version 8.1) [13, 14]. (http://microrna. sanger.ac.uk/sequences/) that have been verified by experiments or predicted by computational methods with high confidence. To ensure that all miRNA hairpins were folded as hairpins, we removed a few of those containing none or more than one RNAfold-predicted hairpin-loop. The positive class used in this work is therefore of 451 miRNA hairpins.

To evaluate our one-class SVM models for miRNA hairpins, we conducted two kinds of experiments.

Cross-validation: like some previous researches $[15,35$, 40], we first prepared the dataset for the cross-validation procedure to compare our method with the other methods. The dataset contained 451 positive examples as described above, and 727 negative examples of miRNAs hairpins. These 727 negative miRNA hairpins were ScorePinhairpins that overlap with the last exon of known codingprotein genes. We randomly partitioned the dataset into three subsets, such that the numbers of both positive and negative examples in each of the three subsets were equivalent or nearly equivalent. Of them, one subset was retrained as the validation data for test prediction methods, and was trained on the two remaining subsets (note that with our method, one-class SVM, only positive examples are used for the training). The cross-validation procedure was repeated three times. Results from three trials were then averaged to produce a single estimation.

Test on chromosomes 10, 15, and 21: we use all known miRNA hairpins, excluding ones on chromosomes 10, 15 and 21, to train the one-class SVM model. This model is then used to recognize miRNA hairpins from ScorePinhairpins (see Section 3.1). Table 1 presents a summary of all data sets used in two kinds of experiments.

Table 1. The data of human miRNA hairpins.

\begin{tabular}{ll}
\hline Experiment & \#Examples \\
\hline Testing on chromosomes & 437 training examples \\
& 41039 hairpin candidates \\
Cross-validation & $2 / 3 \times 451$ training positives \\
& $1 / 3 \times 451$ testing positives \\
& $1 / 3 \times 727$ testing negatives \\
\hline
\end{tabular}

\subsection{One-class support vector machines}

Support vector machine (SVM) is a learning technique based on statistical learning theory [38]. It has been applied to a wide range of real-world tasks. The formulation of SVMs can be considered as a simple linear classification, normally using both negative and positive examples for training. SVMs can perform nonlinear separation by using a kernel technique, which realizes a nonlinear mapping to a feature space. Scholkopf et al. [33] have extended standard SVMs to one-class classification problems. Their approach is to construct a hyperplane that is maximally distant from the origin [33].

In this section, we give details of the algorithm for training one-class SVMs proposed by Scholkopf et al. [33]. The training algorithm is as follows: let the training data $x_{1}, x_{2}, \ldots, x_{l} \in R^{N}$ belong to one class, where $x_{i}$ is a feature vector and $l$ is the number of examples. The oneclass SVM estimates a function that will take the value +1 in a region where the majority of the data points are concentrated, and the value -1 everywhere else [30, 33]. Formally, the function can be written as follows:

$$
f(x)=\left\{\begin{array}{l}
+1 \text { if } x \in S \\
-1 \text { if } x \in \bar{S}
\end{array}\right.
$$

where $S$ is a simple subset of input space and $\bar{S}$ is the complement of $S$. Let $\Phi: X \rightarrow H$ be a kernel map which converts the training examples from the origin space to a feature space. The strategy is to map the data into the feature space corresponding to the kernel, and to separate them from the origin by the maximum margin. In order to separate the data set from the origin, we need to solve the following quadratic programming problem [9, 30, 33]:

$$
\left\{\begin{array}{l}
\min \frac{1}{2}\|w\|^{2}+\frac{1}{v l} \sum_{i=1}^{l} \xi_{i}-\rho \\
\left(w \cdot \Phi\left(x_{i}\right)\right) \geq \rho-\xi_{i}, \quad i=1, \ldots, l ; \xi_{i} \geq 0 .
\end{array}\right.
$$

where $v \in(0,1)$ is a parameter that represents an upper bound on the fraction of outliers in the data, $\rho$ is the margin of the hyperplane with respect to the data, and $x_{i}$ are non-zero slack variables allowing a soft margin. We obtain $w$ and $\rho$ by solving this problem. When we give a new data point $x$ to be classified, a label is assigned ac- 
cording to the decision function, which can be expressed as:

$$
f(x)=\operatorname{sgn}\left(\left(w . \Phi\left(x_{i}\right)\right)-\rho\right)
$$

Instead of solving the primal optimization problem directly, one can consider the following dual program:

$$
\left\{\begin{array}{l}
\max \frac{1}{2} \sum_{i} \alpha_{i} \alpha_{j} K\left(x_{i}, x_{j}\right) \\
0 \leq \alpha_{i} \leq \frac{1}{v l}, \sum_{i} \alpha_{i}=1 .
\end{array}\right.
$$

here, $K\left(x_{i}, x_{j}\right)=\left(\Phi\left(x_{i}, x_{j}\right)\right)$ are kernels, which allow many more general decision functions when the data are not linearly separable, and the hyperplane can be represented in a feature space. The parameters $\alpha_{i}$ are Lagrange multipliers.

In our research, we used the LIBSVM (version 2.84) with three types of kernel functions (linear, polynomial, and radial basis (RBF)). This library is an integrated tool for support vector classification and regression which can handle one-class SVM using the algorithm proposed by Scholkopf et al. [33]. The LIBSVM is available at [43].

\subsection{Structural and sequential features of miRNA hairpins}

There are many miRNA prediction methods which used structural features as key features. However, recent reports have shown that the sequence features are important in predicting miRNA hairpins [39, 40]. Xue et al. [40] indicated that the short contiguous subsequences of miRNA hairpin sequences are significantly distinct from other RNA hairpin sequences. For this reason, we propose a set of features that uses both the sequential features and structural features to characterize the RNA hairpin structure sequences.

For sequential features, we extracted features from RNA hairpin sequences using a 5-nucleotide sliding window along an RNA hairpin sequence, and computed the number of occurrences of each 5-gram. As a result, each sequence is represented by a 1,024-dimensional vector of the number of occurrences of all possible 5-grams. In addition, several other features based on the sequences are considered, such as the number of occurrences of each nucleotide (A, C, G, U) in the 5' and 3' arms and GC- content defined as in [35].

For structural features, we extracted them from the secondary structure of each hairpin. The secondary structures are predicted using RNAfold [16]. The structural features used in our method, were introduced in other previous miRNA prediction methods [15, 35]. The features consist of:

1. miRNA hairpin length as the number of nucleotides.

2. Loop size as the number of unpaired bases in the hairpin loop of the predicted secondary structure.

3. Minimum free energy (MFE) as the total free energy of hairpin structure predicted by using RNAfold tool.

4. Paired bases as the number of nucleotides predicted to be in a hydrogen-bonded state.

5. The numbers of nucleotides from 5' site to the loop start.

6. The number of 2-nt overhangs from 5' site and 3' site to loop start.

In total, the feature vector, which is input to our oneclass SVMs, consists of 1,036 variables. It captures the characteristics of both the sequence and the structure of the RNA hairpin sequences.

\section{RESULTS AND DISCUSSIONS}

\subsection{One-class SVM performance}

We experimentally evaluated our method by using the three-fold cross-validation procedure as described in Section 2.1. In order to avoid miRNA hairpins in the same group (defined in [44]) being divided into different folds, we placed all similar miRNA hairpins in the same fold. Three criteria of precision, recall, and F1-measure were used to evaluate the results. We carried out experiments with three types of kernels (linear, polynomial, and radial basic function (RBF)). For each cross-validation run, we used default parameters $\delta, d$, and various values of parameter $v$ in the range of $[0.07,0.11]$. The prediction results are shown in Table 2 . It can be seen that one-class SVMs worked well with $v=0.10$ and RBF kernels $(\gamma=$ 0.0001 ); the highest F1-measure $=95.27 \%$, precision $=$ $94.63 \%$, and recall $=95.92 \%$. The work in this paper is an extension of our conference paper [37]. Basically, there is one improvement here: we tried to check the contribution of each kind of features to the prediction results.

Table 2. The prediction results of one-class SVMs on the testing dataset. Pre., Rec., and F1. are precision recall and F1-measure, respectively.

\begin{tabular}{cccccccccc}
\hline \multirow{2}{*}{$v$} & \multicolumn{3}{c}{ Linear kernel } & \multicolumn{3}{c}{ Polynomial kernel } & \multicolumn{3}{c}{ RBF kernel } \\
\cline { 2 - 10 } & Pre. & Rec. & F1. & Pre. & Rec. & F1. & Pre. & Rec. & F1. \\
\hline 0.07 & 88.02 & 98.66 & 93.04 & 88.02 & 98.66 & 93.04 & 91.20 & 97.32 & 94.16 \\
0.08 & 89.09 & 98.66 & 93.63 & 89.09 & 98.66 & 93.63 & 94.67 & 95.30 & 94.98 \\
0.09 & 91.03 & 95.30 & 93.11 & 91.03 & 95.30 & 93.11 & 95.27 & 94.63 & 94.95 \\
0.10 & 93.75 & 90.60 & 92.15 & 93.71 & 89.93 & 91.78 & 95.92 & 94.63 & 95.27 \\
0.11 & 94.37 & 89.93 & 92.10 & 93.71 & 89.93 & 91.78 & 95.24 & 93.96 & 94.59 \\
\hline
\end{tabular}


Table 3. The prediction results of one-class SVMs using different feature sets. FS1 is the feature set using only structural features; FS2 is the feature set using both sequential features and structural features; Pre., Rec., and F1. are prediction, recall and F1measure, respectively.

\begin{tabular}{lcccc}
\hline Kernel & $\begin{array}{c}\text { Feature } \\
\text { set }\end{array}$ & Pre. & Rec. & F1. \\
\hline Linear & FS1 & 95.42 & 83.33 & 88.97 \\
& FS2 & 93.75 & 90.60 & 92.15 \\
Polynomial & FS1 & 95.42 & 83.33 & 88.97 \\
& FS2 & 93.71 & 89.93 & 91.78 \\
RBF & FS1 & 92.81 & 86.00 & 89.27 \\
& FS2 & 95.92 & 94.63 & 95.27 \\
\hline
\end{tabular}

To determine the importance of the sequential features introduced for the first time for this research, we removed the sequential features, and then conducted training and testing of the model again. The vector representation of examples using only structural features, denoted as FS1, using two kinds of sequential and structural features, denoted as FS2. Table 3 shows the results of oneclass SVM with the two kinds of vector representations FS1 and FS2 (with the same value for parameter $v=$ 0.10 ). It can be seen that the classifier performance of FS1 is much lower than that of FS2. Therefore, the sequential features are relevant for modeling miRNA hairpins.

We also tried to compare the one-class SVM method with the two-class SVM method, which has been introduced in [35] for the same problem, predicting miRNAs. Different from our one-class SVM method, the two-class SVMs have to be trained on both positive and negative classes of miRNA hairpins. As we mentioned in Section 1 , only positive examples of miRNAs are available, and it is difficult to select some potential miRNA hairpins as "negatives". Similar to some previous researches, we are indisposed to establish a class of 727 "negative” miRNA hairpins as described in Section 2.1, and thus the test results here would be respect for the assumption that these 727 negative examples would be true. Table 4 presents the performance of one-class SVMs and two-class SVMs. It can be seen that although one-class SVMs trained on fewer examples (only positive ones), they performed well when compared with two-class SVM methods.

\subsection{Test on chromosomes 10, 15, and 21}

To emphasize that the one-class SVM is more suitable than a two-class classifier in the problem of recognizing miRNA hairpins, we tested the one-class SVM method on three human chromosomes 10, 15, and 21 and compared the predicted results with the results from the twoclass SVM method described in [35].

In this work, the training dataset is all real miRNA hairpins after excluding ones on the testing chromosomes (Table 1). Through various cross-validation experiments as mentioned in the preceding section, we found that one-class SVM models have a good performance with RBF kernel ( $\gamma=0.0001)$. We fixed these values to build the one-class SVM model for the training dataset of miRNA hairpins in this kind of experiments. We then used ScorePin to scan along both genomic strands of the three chromosomes, 10,15 , and 21 , to find good hairpin candidates. There were 62,508 hairpin candidates with a ScorePin-score $\leq 105$. Among them, 10,035 were confirmed to have an RNAfold-predicted hairpin with a minimum free energy $\leq-25 \mathrm{kcal} / \mathrm{mol}$. Each candidate is represented by a vector of structural and sequential features as described in Section 2.3, and then input to the one-class SVM model. Table 5 shows some predicted miRNA hairpins which have previously been confirmed by labor experiments or other prediction methods. It can be seen, our method recognized all 4 existing miRNA hairpins on chromosome 10, and four of five existing miRNAs on both chromosomes 15 and 21 . Other miRNA hairpins found by our method are provided in the supplementary files (http://www.jaist.ac.jp/ tran/miRNAs/). We also used a two-class SVM method as described in [35] to predict miRNA hairpins on the same chromo somes 10, 15, and 21. In addition to all known miRNA hairpins in the training set of the one-class SVM method, the training data for this two-class SVM model needed negative examples of miRNA hairpins. We got all 727 negative examples of hairpins as described in Section 2.1, together with 437 existing miRNA hairpins in the human genome excluding ones on chromosomes 10,15 , and 21 , to train

Table 4. Comparisons of prediction results between one-class SVMs and two-class SVMs on the testing dataset. FS1 is the feature set using only structural features; FS2 is the feature set using both sequential features and structural features; Pre., Rec., and F1. are prediction, recall, and F1-measure, respectively.

\begin{tabular}{|c|c|c|c|c|c|c|c|}
\hline \multirow{2}{*}{ Feature set } & \multirow{2}{*}{ Kernel } & \multicolumn{3}{|c|}{ One-class SVMs } & \multicolumn{3}{|c|}{ Two-class SVMs } \\
\hline & & Pre. & Rec. & $F 1$. & Pre. & Rec. & $F 1$. \\
\hline \multirow{3}{*}{ FS1 } & Linear & 95.42 & 83.33 & 88.97 & 94.00 & 94.00 & 94.00 \\
\hline & Polynomial & 95.42 & 83.33 & 88.97 & 98.43 & 83.33 & 90.25 \\
\hline & $\mathrm{RBF}$ & 92.81 & 86.00 & 89.27 & 97.76 & 87.33 & 92.25 \\
\hline \multirow{3}{*}{ FS2 } & Linear & 89.09 & 98.66 & 93.63 & 97.96 & 96.64 & 97.30 \\
\hline & Polynomial & 89.09 & 98.66 & 93.63 & 98.63 & 96.64 & 97.63 \\
\hline & $\mathrm{RBF}$ & 95.92 & 94.63 & 95.27 & 97.97 & 97.32 & 97.64 \\
\hline
\end{tabular}


Table 5. The known miRNA hairpins predicted by one-class SVMs on chromosomes 10, 15, and 21. Location consists of the start point and end point of the miRNA hairpin on the chromosome. MFE is a minimum free energy of the miRNA hairpin structure.

\begin{tabular}{cclc}
\hline $\begin{array}{c}\text { Chr } \\
\#\end{array}$ & \multicolumn{1}{c}{ Location } & miRNA_ID & MFE \\
\hline \multirow{2}{*}{10} & $17927110: 17927200$ & hsa-mir-511-1 & -34.6 \\
& $17927110: 17927200$ & hsa-mir-511-2 & -34.6 \\
& $52729335: 52729425$ & hsa-mir-605 & -54.8 \\
& $104186251: 104186341$ & hsa-mir-146b & -41.2 \\
\hline \multirow{2}{*}{15} & $60903206: 60903296$ & hsa-mir-190 & -32.5 \\
& $86956075: 86956165$ & hsa-mir-7-2 & -43.1 \\
& $77289181: 77289271$ & hsa-mir-184 & -37.9 \\
& $87712251: 87712341$ & hsa-mir-9-3 & -41.1 \\
\hline \multirow{2}{*}{21} & $16833274: 16833364$ & hsa-mir-99a & -47.0 \\
& $25868151: 25868241$ & hsa-mir-155 & -39.5 \\
& $36014883: 36014973$ & hsa-mir-802 & -35.0 \\
& $16834016: 16834106$ & hsa-let-7c & -43.2 \\
\hline
\end{tabular}

Table 6. The known miRNA hairpins predicted by two-class SVMs on chromosomes 10, 15, and 21. Location consists of the start point and end point of the miRNA hairpin on the chromosome. MFE is a minimum value of the miRNA hairpin structure.

\begin{tabular}{cccc}
\hline Ch & \multicolumn{1}{c}{ Location } & \multicolumn{1}{c}{ miRNA_ID } & MFE \\
\hline \multirow{2}{*}{10} & $17927110: 17927200$ & hsa-mir-511-1 & -34.6 \\
& $17927110: 17927200$ & hsa-mir-511-2 & -34.6 \\
& $52729335: 52729425$ & hsa-mir-605 & -54.8 \\
& $104186251: 104186341$ & hsa-mir-146b & -41.2 \\
\hline \multirow{2}{*}{15} & $60903206: 60903296$ & hsa-mir-190 & -32.5 \\
& $86956075: 86956165$ & hsa-mir-7-2 & -43.1 \\
\hline \multirow{2}{*}{21} & $16833274: 16833364$ & hsa-mir-99a & -47.0 \\
& $25868151: 25868241$ & hsa-mir-155 & -39.5 \\
& $36014883: 36014973$ & hsa-mir-802 & -35.0 \\
\hline
\end{tabular}

the discriminative two-class SVM model. Table 6 shows some miRNA hairpins predicted by the two-class SVM model. Among them, all four miRNA hairpins on chromosome 10 were identified as same as using the one-class SVM. Consistent with the results reported in [35], the two-class SVM also recognized three of five existing miRNA hairpins on chromosome 21, and two of four on chromosome 15. Especially, while one-class SVM recognized correctly an additional miRNA hairpin on chromosome 21, the two-class SVM predicted them as negatives. The reasons why two-class SVM method incorrectly recognized some known miRNA hairpins might be that the two-class SVM training is based on some negative examples of miRNA hairpins, which might not be true due to the way to select "negative" ones.

\section{CONCLUSIONS}

We have introduced a one-class learning method to predict pre-miRNAs in the human genome. Our one-class support vector machine method has an advantage over other two-class discriminative models: it uses only available positive examples of miRNA hairpins for building the model, while all existing methods for the same problem must use additional negative ones, which are not available, since it is hard to find true negatives for the training of a two-class classifier. Our method showed good performance, and we have illustrated the case of testing on chromosomes 10, 15 and 21, in which our method gave the prediction results more precise than those from an existing two-class support vector machine method.

\section{ACKNOWLEDGMENTS}

The research described in this paper was partially supported by the Institute for Bioinformatics Research and Development of the Japan Science and Technology Agency, and by COE project JCP KS1 of the Japan Advanced Institute of Science and Technology. The first author has been supported by Japanese government scholarship (Monbukagakusho) to study in Japan. The authors also would like to thank Prof. Ivo Hofacker from University of Vienna for providing the ViennaRNA package and Dr. Chih-Jen Lin from National Taiwan University for providing the LIBSVM tool.

\section{REFERENCES}

[1] V. Ambros. (2004) The functions of animal microRNAs. Nature, 431 350-355.

[2] D. P. Bartel. (2004) MicroRNAs: genomics, biogenesis, mechanism, and function. Cell, 116, 281-297.

[3] I. Bentwich. (2005) Prediction and validation of microRNAs and their targets. FEBS Lett, 579, 5904-5910.

[4] E. Berezikov, E. Cuppen, and R. H. Plasterk. (2006) Approaches to microRNA discovery. Nat. Genet., 38, S2-S7.

[5] C. J. Burges. (1998) A tutorial on support vector machines for pattern recognition. J. Data Mining and Knowledge Discovery, 2, 121-167.

[6] M. T. Bohnsack, K. Czaplinski and D. Grlich. (2004) Exportin 5 is

a RanGTP-dependent dsRNA-binding protein that mediates nuclear export of pre-miRNAs. RNA, 10, 185-191.

[7] J. Brown, P. Sanseau. (2005) A computational view of microRNAs and their targets. Drug discovery today: biosilico, 10(8), 595-601.

[8] C. -C. Chang, and C. -J. Lin. (2001) LIBSVM: a library for support vector machines.

[9] Y. Chen, X. Zhou, and T. S. Huang. (2001) One-class SVM for learning in image retrieval. Proc. IEEE Int'l Conf. on Image Processing, Thessaloniki, Greece.

[10] M. A. Denli, B. J. Tops, H. A. Plasterk, R. F. Ketting, and G. J.Hannon. (2004) Processing of primary microRNAs by the Microprocessor complex. Nature, 432, 231-235.

[11] Y. Grad, J. Aach, G. D. Hayes, B. J. Reinhart, G. M. Church, G. Ruvkun, and J. Kim. (2003) Computational and experimental identification of C. elegans microRNAs. Mol Cell, 11,1253-1263.

[12] R. I. Gregory, K. P. Yan, G. Amuthan, T. Chendrimada, B. Doratotaj, N. Cooch, and R. Shiekhattar. (2004) The microprocessor complex mediates the genesis of microRNAs. Nature, 423, 235-240.

[13] S. Griffiths-Jones, R. J. Grocock, S. Dongen, A. Bateman, A. J. Enright. (2006) miRBase: microRNA sequences, targets and gene nomenclature. Nucleic Acids Res., 34, D140-D144.

[14] S. Griffiths-Jones. (2004) The microRNA Registry, Nucleic Acids Res., 32, D109-D111.

[15] S. A. Helvik, O. S. Jr, and P. Strom. (2007) Reliable prediction of Drosha processing sites improves microRNA gene prediction. Bioinfor- 
matics, 23(2), 142-149.

[16] I. L. Hofacker, S. Fontana, W. Stadler, S. Bonhoeffer, M. Tacker, and P. Schuster. (1994) Fast folding and comparison of RNA secondary structures, Monatshefte f. Chemie, 125, 167-188.

[17] I. L. Hofacker. (2003) Vienna RNA secondary structure server. Nucleic Acids Res, 31, 3429-3431.

[18] M. Kiriakidou, P. T. Nelson, A. Kouranov, P. Fitziev, C. Bouyioukos, Z. Mourelatos, and A. Hatzigeorgiou. (2004) A combined computational experimental approach predicts human microRNA targets. Genes Dev, 18, 1165-1178.

[19] A. Kowalczyk, and B. Raskutti. (2002) One-class svm for yeast regulation prediction. Proc. SIGKDD Explorations Workshop, 99-100.

[20] R. Kohavi. (1995) A study of cross-validation and bootstrap for accuracy estimation and model selection. Proc. 14th IJCAI, San Francisco, CA, Morgan Kaufmann Pubshers, 1137-1143,.

[21] Y. Kong, J.-H. Han. (2005) MicroRNA: Biological and computational perspective. Geno. Prot. Bioinfo., 3(2), 62-72.

[22] J. Krol, K. Sobczak, U. Wilcztnska, M. Drath, A. Jasinska, D.

Kaczynska, and W. J. Krzyzosiak. (2004) Structural features of microRNA (miRNA) precursors and their relevance to miRNA biogenesis and small interfering RNA/short hairpin RNA design. J Biol Chem, 279, 42230-42239.

[23] M. Lagos-Quintana, R. Rauhut, W. Lendeckel, and T. Tuschl. (2001) Identification of novel gene coding for small expressed RNAs. Science, 294, 853-858.

[24] E. C. Lai, P. Tomancak, R. W. Williams, and G. M. Rubin. (2003) Computational identification of Drosophila microRNA genes. Genome Biol, 4, R42.

[25] Y. Lee, C. Ahn, J. Han, H. Choi, J. Yim, P. Provost, O. Radmark, S Kim, and V. N. Kim. (2003) The nuclear RNase III Drosha initiates microRNA processing. Nature, 424, 415-419.

[26] Y. Lee, M. Kim, J. Han, K. Yeom, S. H. Lee, S. H. Baek, and V. N. Kim. (2004) MicroRNA genes are transcribed by RNA polymerase II. EmboJ, 23,4051-4060.

[27] L. P. Lim, M. E. Glasner, S. Yekta, C. B. Burge, and D. P. Bartel. (2003) Vertebrate microRNA genes. Science, 299, 1540.

[28] L. P. Lim, N. C. Lau, E. G. Weinstein, A. Abdelhakim, S. Yekta, M. W. Rhoades, C. B. Burge, and D. P. Bartel. (2003) The microRNAs of Caenorhabditis elegans. Genes Dev, 17, 991-1008.

[29] E. Lund, S. Guttinger, A. Calado, J. E. Dahlberg, and U. Kutay. (2004) Nuclear export of microRNA precursors. Science, 303, 95-98.

[30] L. M. Manevitz, and M. Yousef. (2001) One-class SVMs for document classification. Journal of Machine Learning, 2, 139-154.
[31] J. W. Nam, K. R. Shin, Y. V. Lee, N. Kim, and B. T. Zhang. (2005) Human microRNA prediction through a probabilistic co-learning model of sequence and structure. Nucleic Acids Res., 33, 3570-3581.

[32] U. Ohler, S. Yekta, L. P. Lim, D. P. Bartel, and C. B. Burge. (2004) Patterns of flanking sequence conservation and a characteristic upstream motif for microRNA gene identification. RNA, 10, 1309-1322.

[33] B. Scholkopf, J. C. Platt, J. Shawe-Taylor, A. J. Smola, and R. C. Williamson. (2001) Estimating the support of a high-dimensional distribution. Neural Comput, 13, 1443-1471.

[34] P. Strom, O. S. Jr, M. Nedland, T. B. Grnfeld, Y. Lin, M. B.

Bass, J. Canon. (2006) Conserved microRNA characteristics in mammals. Oligonucleotides, 16, 115-144.

[35] K. Szafranski, M. Megraw, M. Reczko, G. H. Hatzigeorgiou. (2006) Support vector machine for predicting microRNA hairpins. Proc. The 2006 International Conference on Bioinformatics and Computational Biology, 270-276.

[36] A. Tsirigos, and I. Rigoutsos. (2005) A sensitive, support-vectormachine method for the detection of horizontal gene transfers in viral, archaeal and bacterial genomes. Nucleic Acids Research, 33(12):36993707.

[37] D. H. Tran, T. H. Pham, K. Satou, and T. B. Ho. (2008) Prediction of microRNA hairpins using one-class support vector machine. Proc. The $2^{\text {nd }}$ international conference on bioinformatics and biomedical engineering (iCBBE), Sanghai, China, May 16-18.

[38] V. Vapnik. Statistical learning theory, Wiley, Chichester, United Kingdom, 1998.

[39] X. Xie, J. Lu, E. J. Kulbokas, T. R. Golub, V. Mootha, K. LindbladToh, E. S. Lander, and M. Kellis. (2005) Systematic discovery of regulatory motifs in human promoters and 3' UTRs by comparison of several mammals. Nature, 434, 338-346.

[40] C. Xue, F. Li, T. He, G. P. Liu, Y. Li, and X. Zhang. (2005) Classification of real and pseudo microRNA precursors using local structuresequence features and support vector machine. BMC Bioinformatics, 6, 310.

[41] L. H. Yang, W. Hsu, M. L. Lee, and L. Wong. (2006) Identification of microRNA precursors via SVM, Proc. The 4th AsiaPacificBioinformatics Conference, 267-276.

[42] Y. Zeng, R. Yi, and B. R. Cullen. (2005) Recognition and cleavage of primary microRNA precursors by the nuclear processing enzyme Drosha. Embo J, 24,138-148.

[43] http://www.csie.ntu.edu.tw/ cjlin/libsvm/

[44] http://microrna.sanger.ac.uk/sequences/index.shtml

[45] http://www.tbi.univie.ac.at/RNA/ 